\title{
BUENOS TIEMPOS PARA EL NEOCONSTITUCIONALISMO (SOBRE LA APARICIÓN DE EL EXAMEN DE PROPORCIONALIDAD EN EL DERECHO CONSTITUCIONAL)*
}

\section{GOOD TIMES FOR NEO-CONSTITUTIONALISM (ON THE APPEARANCE OF THE EXAM OF PROPORTIONALITY IN CONSTITUTIONAL LAW)}

Leonardo García Jaramillo**

* Laura Clérico. El examen de proporcionalidad en el derecho constitucional. 408 págs. Eudeba. Buenos Aires. (2009).

** Universidad EAFIT-Medellín, Departamento de Humanidades. Diálogos personales y epistolares con Miguel Carbonell, Rodolfo Arango y Carlos Bernal Pulido, además de algunos con Laura ClÉRICO, me han permitido formar un mejor entendimiento de la cuestión sobre la que me ocupo aquí. Conste mi sincero agradecimiento. 
El lustro que concluye este año ha sido determinante para el neoconstitucionalismo. Desde 1997 cuando la profesora italiana Susanna Pozzolo acuñara el término en una conferencia impartida en la XVIII versión del Internationale Vereinigung für Rechts-und Sozialphilosophie (IVR) celebrado en Buenos Aires, no sólo los autores que forjan el canon (Dworkin, Alexy, Ferrajoli, Zagrebelsky) han publicado importantes trabajos en los que responden a las críticas que se les han dirigido desde diversos enfoques de la ciencia jurídica, depuran aspectos de sus teorías y precisan elementos del andamiaje conceptual en el que se estructura, sino que en nuestro idioma han aparecido valiosos trabajos durante tal período'.

$\mathrm{Si}$ alguna característica trasversal pudiera predicarse de tales libros, señalaría un cometido común de sus autores por explicitar y contribuir así a estructurar el neoconstitucionalismo como canon dominante en la teoría constitucional contemporánea, con hondas repercusiones en la concepción, práctica y enseñanza del derecho. La forma en la cual se acomete dicho reto es dotando de mayor solidez sus estructuras medulares, es decir, las instituciones jurídicas, los elementos doctrinarios, los planteamientos teóricos y las herramientas interpretativas que desde mediados de la segunda mitad del siglo pasado comenzaron a irradiar de forma determinante al derecho constitucional y al derecho ordinario en los países europeos y, luego, en diversos contextos latinoamericanos. De forma significativa se pretende receptar en dichos trabajos las estructuras medulares en contextos determinados donde los procesos de cambio constitucional han estado acompañados por drásticos giros jurisprudenciales que, entre otras características, se destacan por acercar el texto constitucional a los ciudadanos y por sofisticar los mecanismos de control jurisdiccional de los actos públicos y privados que inciden en la órbita de los derechos fundamentales.

1 Por mencionar algunos de los principales: Miguel Carbonell (ed.). Neoconstitucionalismo(s). Madrid. Trotta. $4^{\mathrm{a}}$ ed. (2009); (ed.) Teoría del neoconstitucionalismo. Madrid. Trotta. (2007). CARLos Bernal Pulido. El principio de proporcionalidad y los derechos fundamentales. Madrid. Centro de Estudios Constitucionales. $3^{\text {a }}$ ed. (2007); El derecho de los derechos. Bogotá. Universidad Externado. (2005); El neoconstitucionalismo y la normatividad del derecho. Bogotá. Universidad Externado. (2009). Rodolfo Arango. El concepto de derechos sociales fundamentales. Bogotá. Legis. (2005). Diego López Medina. El derecho de los jueces. Bogotá. Legis. $2^{\mathrm{a}}$ ed. (2006). Daniel Bonilla - MaNuel IturRalde (eds.). Hacia un nuevo derecho constitucional. Bogotá. Universidad de los Andes. (2005). Gloria Lopera Mesa. Principio de proporcionalidad y ley penal. Madrid. Centro de Estudios Constitucionales. (2006). LuIs Prieto SAnchís. Justicia constitucional y derechos fundamentales. Madrid. Trotta. (2003). Manuel Atienza, Josep Aguiló \& Juan Ruiz Manero. Fragmentos para una teoría de la Constitución. Madrid. Iustel. (2007). Luis Roberto BARroso. El neoconstitucionalismo y la constitucionalización del derecho. México D.F. UNAM-IIJ. (2008). Víctor Ferreres. Justicia constitucional y democracia. Madrid. Centro de Estudios Constitucionales. $2^{\mathrm{a}}$ ed. (2007). RoBerto Gargarella. Teoría y crítica del derecho constitucional (coord.). Buenos Aires. Abeledo-Perrot. 2 vols. (2008). José Juan Moreso. Constitución. Modelo para armar. Barcelona. Marcial Pons. (2009). Alfonso García Figueroa. Criaturas de la moralidad. Una aproximación neoconstitucionalista al derecho a través de los derechos. Madrid. Trotta. (2009). Miguel Carbonell - Leonardo García JARAMILLO (eds.). El canon neoconstitucional. Bogotá. Universidad Externado. (2010). 
No es este el momento para detenerse en una apología al neoconstitucionalismo ni para realizar una reconstrucción de sus estructuras medulares. Hay que señalar, sin embargo, que el principio de proporcionalidad como estructura argumentativa para controlar las restricciones en derechos fundamentales que resultan constitucionalmente ilegítimas, ineficaces para alcanzar el objetivo previsto o que suponen un sacrificio desproporcionado de los mismos, constituye no sólo una de tales estructuras que hacen parte del canon neoconstitucional, sino una de las que mayor atención académica ha recibido, con significativo influjo doctrinario y jurisprudencial en diversas latitudes ${ }^{2}$.

No obstante hallarse su creación en el ya extinto derecho de policía prusiano, la proporcionalidad (principio, mandato, regla o criterio) se ha convertido desde mediados del siglo pasado en una herramienta cada vez más utilizada en diversas áreas del derecho para solucionar los conflictos que se presentan cuando en un caso concreto que vincula derechos o fines constitucionalmente protegidos, concurren varias soluciones posibles de las que naturalmente sólo puede elegirse una por la estructura misma de los principios. De este punto queda claro que en el derecho constitucional es donde su utilización ha sido más generalizada y, por tanto, donde mayor sofisticación ha adquirido su formulación. Interesantes experiencias sobre la aplicación jurisprudencial de la proporcionalidad se encuentran en países como Alemania, España, Italia, Estados Unidos, México, Colombia y Perú3.

En su formulación procedente de la dogmática alemana, al principio de proporcionalidad en sentido amplio lo integran tres subprincipios: adecuación o idoneidad, con el cual se verifica que la medida enjuiciada que limita un derecho o bien constitucionalmente protegido, sea adecuada para contribuir al logro de un fin constitucionalmente legítimo; necesidad, dirigido a establecer si la medida enjuiciada es la más benigna con el derecho fundamental afectado, entre todas aquellas que sean igualmente idóneas para alcanzar el fin perseguido por la intervención; la proporcionalidad en sentido estricto, consiste

2 Quizás el principal antecedente disponible en nuestro idioma sea la Teoría de los derechos fundamentales de AleXY, que en el 2007, conoció una segunda edición en traducción de CARLos BerNAL Pulido.

3 Ejemplos y análisis del país germano con respecto a la proporcionalidad en diferentes ámbitos jurídicos, se encuentran en LAURA CLÉRICO. El examen de proporcionalidad en el derecho constitucional. $O p$. cit. Acerca del recurso a la proporcionalidad en los otros países, ver los estudios reunidos en Miguel Carbonell (ed.). El principio de proporcionalidad y la interpretación constitucional. Op. cit. Para el caso español, ver, Carlos Bernal Pulido. El principio de proporcionalidad y los derechos fundamentales. Op. cit. Daniel SARmiento Ramírez. El principio de proporcionalidad en el derecho administrativo. Un análisis desde el derecho español. Bogotá. Universidad Externado. (2007). IsABEL Perelló D. "El principio de proporcionalidad y la jurisprudencia constitucional". En Jueces para la democracia $\mathrm{n}^{\circ}$ 28. (1997). Un interesante análisis crítico de la aplicación del principio por parte del Tribunal Constitucional Peruano, se encuentra en Carlos Bernal Pulido. "La aplicación del principio de proporcionalidad en el juicio de igualdad". En El neoconstitucionalismo y la normatividad del derecho. Op. cit. Capítulo 11. 
en un juicio en el cual se pondera la intensidad del sacrificio del derecho con la importancia que reviste en el caso concreto el logro de la finalidad que se busca satisfacer con su limitación.

Estos subprincipios son aplicados de forma conjunta y escalonada, conformando así un rumbo argumentativo que, al aplicarse lexicográficamente, orienta el control de constitucionalidad de las actuaciones del poder público que inciden en la órbita de los derechos fundamentales. Se imponen dos cuestiones que deben resolverse en el examen de constitucionalidad en sede del submandato de idoneidad, pues primero se considera la legitimidad del fin que persigue la medida, y luego que ella sea idónea, es decir, eficaz para alcanzar el objetivo que se propone. Un fin legislativo es definido por CLÉRICO como un estado de cosas que debe alcanzarse y que tiene que ver, bien con el cumplimiento de un derecho fundamental o de un bien colectivo, o bien con evitar que estos objetos sean restringidos de manera gravosa. Sobre la necesidad, CLÉRICO realiza su análisis a partir de la distinción entre dos exigencias, a saber, para concluir que una medida legislativa no supera el examen del mandato de necesidad -por lo que es declarada inconstitucional-, al menos otro medio alternativo debe revestir de una idoneidad equivalente para propender por la obtención de su fin inmediato; y sobre la segunda exigencia, una medida legislativa no supera el examen del mandato de necesidad cuando alguno de los medios alternativos que reviste siquiera de idoneidad equivalente interviene además en un menor grado en el derecho fundamental afectado, es decir, se determina si alguno de los medios alternativos no afecta negativamente al derecho fundamental. Y sobre la ponderación se trata entonces de establecer si la afectación del derecho fundamental resulta justificada por la importancia de la realización del fin que con ella se persigue, lo que supone llevar a cabo una ponderación entre el peso de las razones a favor y en contra de la constitucionalidad de la medida enjuiciada.

La plausibilidad iusconstitucional de la limitación a un derecho se determina precisamente en la medida en que se superen las exigencias impuestas por el principio de proporcionalidad en sentido amplio. La herramienta en la que se constituye este principio para justificar la limitación sirve al intérprete para determinar, en primer lugar, el bien jurídico protegido que es limitado, el acto mismo (por acción u omisión ${ }^{4}$ ) que interviene en el derecho y los límites a la limitación del derecho que sufre la restricción.

En este sentido es valioso recalcar que se aclara la necesidad de observar dos "costados" con respecto a la validez de un derecho: uno pasivo y otro activo. La admisibilidad constitucional de una medida que afecta un derecho determinado, se establece al precisar que desde el primer costado los derechos

4 Con mayor precisión, como se estudia en el último capítulo, la limitación a un derecho puede acontecer por acción excesiva pero también por acción insuficiente o por omisión. 
pueden ser limitados en la medida en que valen en el marco de un conjunto de otras normas, por lo que su concreción práctica debe estar mediada por la validez del otro derecho en contraposición. Desde el segundo costado la validez de un derecho que se limita comporta el que los derechos actúan como límites pero que así mismo ponen restricciones a su limitación, lo cual admite que la validez de un derecho fundamental eleva una pretensión de ejercicio.

En el último capítulo se estudia dicho elemento doctrinario que no ha merecido tanta atención por el estado del arte, pues la aplicación de los tres submandatos de la proporcionalidad, en sentido amplio, se han analizado fundamentalmente desde un enfoque en la prohibición de restringir excesivamente un derecho, mientras que se había dejado un tanto de lado hasta la aparición de la investigación de CLÉRICO el análisis de la aplicación del mandato de proporcionalidad desde un enfoque en la prohibición constitucional por omisión o acción insuficiente o deficiente. Las razones de este descuido doctrinario son analizadas en el referido capítulo.

Dentro del iusconstitucionalismo, la utilización de la proporcionalidad ha estado fundamentalmente enfocada en el control de la actividad de los poderes públicos que de uno u otro modo incide de forma leve, media o grave en la garantía y la realización de los derechos fundamentales. Al utilizar la conocida escala formulada por ALEXY para señalar los tres potenciales grados de afectación de un principio que contiene un derecho, queda claro, de igual forma, que la proporcionalidad es también una herramienta que ha mostrado su utilidad al permitir determinar el alcance de los derechos fundamentales, tanto en sí mismos cuando se debate un caso entre el ciudadano y el Estado, como cuando los derechos se debaten en sede horizontal, es decir, y sobre todo gracias a la reciente dogmática de los derechos fundamentales, con respecto a las relaciones entre particulares. En el papel del control constitucional de las intervenciones legislativas en los derechos fundamentales ha estado enfocado el desarrollo doctrinal del principio de proporcionalidad ${ }^{5}$.

Además de presentar a la comunidad académica-jurídica un nuevo material bibliográfico, la motivación que inspira estas líneas es la de celebrar el acontecimiento que supone la esperada aparición de la versión castellana del libro de la profesora bonaerense LAURA Clérico. El examen de proporcionalidad en el derecho constitucional corresponde a la traducción de la tesis doctoral que defendiera en la Universidad de Kiel (Alemania) en el año 2000 y la cual fue dirigida por Robert Alexy. La versión original se tituló Die Struktur der Verhältnismäßigkeit (La estructura de la proporcionalidad) y fue publicada en la reputada editorial Nomos, de Baden-Baden, un año después de que se defendiera. Dicho trabajo ganó el premio a la mejor tesis

5 Gloria Lopera Mesa. Principio de proporcionalidad y ley penal. Op. cit. Capítulo primero. II. 
doctoral que otorga la Asociación de Doctores de Derecho de Kiel (Kieler Doctores Iuris E.V.).

Por la complejidad propia del principio de proporcionalidad, debido esencialmente a los diversos aspectos que vinculan su análisis (función, estructura, fundamento constitucional, racionalidad y presupuestos de aplicación) y a los elementos directamente relacionados con su aplicación (el grado de legitimidad de los tribunales constitucionales para aplicarlo y el grado de racionalidad propio del examen), realizar una crítica al libro es un asunto que está fuera de mis posibilidades. El cometido de esta reseña es, por tanto, sintetizar los principales aspectos del libro y enmarcar su utilidad dentro del canon neoconstitucional.

Lo primero que hay que decir del libro es que su título designa con precisión su tema. El examen de proporcionalidad en el derecho constitucional se compone de una introducción y cuatro capítulos. Analiza con rigurosa erudición los tres subprincipios del principio de proporcionalidad en sentido amplio, respectivamente, en los tres primeros capítulos. En el cuarto, reconstruye algunos elementos de los tres subprincipios previamente estudiados para evaluarlos con respecto a los mandatos de prohibición frente al exceso de restricción (übermaßverbot), y de prohibición por omisión, defecto o acción insuficiente (untermaßverbot $)^{6}$.

En un Excurso al primer capítulo se sustenta la importancia de distinguir entre los casos claros y dudosos de la ilegitimidad del fin que procura alcanzar la medida que interviene en un derecho fundamental. El examen constitucional de la legitimidad del medio planteado para conseguir un objetivo determinado y el examen constitucional del fin que se constituye en el objetivo (que se realiza en sede del primer subprincipio: de idoneidad), son exámenes independientes porque se pueden determinar sin ponderar los principios que colisionan. Sin tener que ponderar en los casos fáciles es posible determinar la legitimidad o no del fin, ya que puede subsistir una prohibición definitiva del fin que persigue la intervención legislativa, o expresamente aparece una no-prohibición definitiva del mismo. La prohibición prima facie del fin legislativo se presenta en los casos difíciles porque hay una colisión del mismo con otros principios que secundan su legitimidad. Para determinar si la prohibición prima facie se vuelve definitiva hay que ponderar el primer tipo de prohibición con los otros principios constitucionales. Es decir, si el operador se encuentra ante un caso fácil puede determinar la legitimidad o no del fin, prescindiendo del examen de proporcionalidad en sentido estricto, ya que, o hay una prohibición definitiva del fin que persigue la intervención legislativa, o se revela palmariamente una no-prohibición del mismo. Debe

6 Dicho capítulo, con idéntico título "El examen de proporcionalidad: entre el exceso por acción y la insuficiencia por omisión o defecto" se había publicado en español previamente. Cfr.: Miguel CARBONELL (ed.). El principio de proporcionalidad y la interpretación constitucional. Op. cit. Capítulo $5^{\circ}$. 
considerarse entonces la dificultad del caso para determinar la necesidad de ponderar entre la prohibición constitucional prima facie del fin del legislador y los otros derechos o fines constitucionalmente protegidos que hablan en favor de su legitimidad. La prevalencia definitiva de uno de los dos principios en pugna se precisa al ponderar entre la prohibición prima facie y los otros principios. En los casos difíciles entonces la ponderación permite saber si la prohibición prima facie prevalece y se vuelve una prohibición definitiva, o si por el contrario, los principios que se le oponen prevalecen y dan lugar a una no-prohibición definitiva del fin perseguido por el legislador.

En un Excurso al tercer capítulo se plantean algunas objeciones a la ponderación en el marco del tercer subprincipio de proporcionalidad. De gran importancia este aparte dentro de la estructura del libro porque la ponderación orientada por reglas se presenta como un modelo que resiste fundamentales críticas que se han enderezado en contra del modelo exclusivamente abstracto de la ponderación. El mecanismo metodológico para resolver colisiones entre derechos fundamentales (que constituyen el mejor ejemplo de los principios jurídicos en las constituciones contemporáneas) que encierra la ponderación, debe entenderse conforme a CLÉRICO, como un mecanismo que no resulta excluyente de la subsunción, sino complementario en el complejo proceso de interpretar, aplicar y desarrollar el derecho.

A dicho modelo se llega luego de reconstruir la estructura de cada uno de los tres submandatos de la proporcionalidad en sentido amplio y de distinguir con respecto a estas la intensidad de aplicación de cada submandato. El modelo orientado por reglas aproxima a la resolución por el problema de la racionalidad de la ponderación. Al examen de proporcionalidad en sentido amplio lo integran tres subprincipios, de los cuales el último es la ponderación o proporcionalidad en sentido estricto. A su vez la ponderación se estructura a partir de tres elementos, a saber, la ley de la ponderación, la fórmula del peso y la carga de argumentación. La ley de la ponderación permite determinar relaciones prioritarias que posibilitan resolver las situaciones de colisión entre principios.

Concluye el libro con un anexo que contiene una selección de las reglas y orientaciones argumentativas que se plantearon y sustentaron en los cuatro capítulos. Dividen el anexo cinco grupos de reglas y orientaciones: para el examen de la justificación iusfundamental de la limitación de los derechos, las propias del mandato de idoneidad, del mandato de necesidad y de la ponderación; finalmente, una formulación general y unas reglas del examen de prohibición por omisión o acción insuficiente como examen de proporcionalidad en sentido amplio.

Se analizan de forma estructurada las reglas que resultan de la ponderación, las cuales tienen efectos como aquellos propios de los precedentes. En contextos de práctica jurisprudencial a dichas reglas que son resultado de la 
ponderación se les conoce, siguiendo a AleXY, como normas adscriptas; en Colombia han sido denominadas no pacíficamente ${ }^{7}$ subreglas. Como principio de racionalidad en la aplicación del derecho, la posibilidad de generalización requiere que se conformen este tipo de reglas que desencadenan efectos precedentes. La argumentación a partir de estas reglas comporta riesgos y ventajas que se estructuran en el capítulo sobre el tercer subprincipio de la proporcionalidad. La importancia de esta cuestión radica en que en los resultados de la ponderación quedan reglas que conforman una red cuyo rigor en la aplicación queda el que sea una red sin fisuras. Las reglas resultado de la ponderación elevan entonces una pretensión de aplicabilidad ante casos iguales. La importancia que reitera CLÉRICO de la práctica jurisprudencial medianamente estandarizada tiene trascendental relación con esta idea, pues cada regla va configurando la red, que tiene efectos jurídicos idénticos a las leyes aprobadas por el congreso. Sobra destacar la relevancia de esta cuestión para la agenda neoconstitucional, en particular en el proceso de desbancar a la ley como única fuente de derecho.

La propia autora se encarga de relevar a quien pretenda argumentar la adscripción del libro al neoconstitucionalismo. No sólo su cometido investigativo hace parte de uno de los elementos medulares de las nuevas doctrinas sobre el constitucionalismo, sino que desde la introducción se enmarca dicha cuestión. En razón del libro resultan relevantes dos características del neoconstitucionalismo y, en particular, del tipo de constituciones que desde mediados del siglo pasado se promulgaron en Europa con pronta repercusión en Latinoamérica, a saber, la positivización de los derechos humanos y la pretensión de garantizarlos y posibilitar su ejercicio como elementos distintivos de la legitimidad del poder estatal. Estas dos características comportan cuestiones correlativas a la justificabilidad iusfundamental, es decir, la admisibilidad constitucional de las decisiones sobre derechos fundamentales, la interpretación de su alcance, las determinaciones de los legitimados para interpretarlos y, finalmente, los límites que se deben imponer a la limitación de un derecho por cuenta de la realización de otro derecho.

La diáfana estructura del libro guía al estudioso hacia el centro del trabajo. La reconstrucción del examen de proporcionalidad en sentido amplio desde la perspectiva de la jurisprudencia del Tribunal Constitucional Federal Alemán, no se reduce, sin embargo, a una reconstrucción ni a un mero análisis de la forma en la cual dicho Tribunal ha enfrentado el desafío que

7 Bernal Pulido ha sostenido que no parece apropiada la designación porque se puede dar la errada impresión de que este tipo de normas son reglas implícitas en otras reglas o una clase de normas de inferior jerarquía con respecto a la fuente del derecho en la que se encuentra la disposición a la que se adscribe. La norma adscrita, o subregla, no es inferior - se sustenta- porque al ser concreción de las disposiciones constitucionales o legales posee el carácter propiamente de regla o de principio, pero en cualquier asunto tiene rango constitucional o legal, según el caso, para resolver casos futuros. Véase su libro El derecho de los derechos. Op. cit. Págs. 213-214 y 260. 
impone la práctica de la aplicación de los derechos fundamentales en los casos difíciles, antes bien, como destaca Bernal Pulido, el libro de Clérico constituye sin duda el análisis más refinado de la estructura del principio de proporcionalidad que hasta el momento se conoce ${ }^{8}$. El libro aporta elementos importantes al desarrollo de las exigencias de claridad conceptual que, con respecto a la proporcionalidad en sentido amplio, impone la dogmática constitucional contemporánea.

A la ponderación se le critica por irracional (crítica metodológica) y porque carece de una dogmática que se refiera a la aplicación de cada uno de los derechos fundamentales (crítica dogmática). Además, desde el punto de vista político, se objeta dicho mecanismo metodológico porque supone la ampliación de las competencias jurisprudenciales en materia de configuración de los derechos, lo cual va en detrimento de las competencias del parlamento9. CLÉRICO, precisamente, parte de reconocer la admisibilidad de la segunda crítica para reconstruir una dogmática regida por reglas. No repudia una teoría general de la ponderación (o modelo exclusivamente abstracto) pero se muestra más favorable a la creación de reglas precisas para cada uno de los derechos; esto responde a la segunda objeción y, al ahondar en una dogmática específica referida a la ponderación de cada uno de los derechos fundamentales, se debilitan las objeciones primera y tercera.

En este sentido puede afirmarse que el intento del libro es reconstruir los tres subprincipios del examen de proporcionalidad en sentido amplio, como un examen orientado por reglas. Este critica los dos esquemas extremos de este modelo, ya que ni el primero para el que no se aplican reglas sino que sólo se pondera, ni el segundo que no admite ponderación, permiten reconstruir una práctica constitucional estable. Por eso se prefiere el modelo de ponderación orientado por reglas. De igual forma, subyace a la argumentación de CLÉRICo la tensión inmanente entre la validez concreta del derecho que requiere la mayor consideración posible de las circunstancias concretas del caso y la vinculación a las reglas que orientan el examen, las cuales a su vez tienen que justificarse para no convertir la aplicación del examen en automática y

8 Carlos Bernal Pulido. El principio de proporcionalidad y los derechos fundamentales. Op. cit. Capítulo sexto. II.

9 Carlos Bernal Pulido ha rebatido estas críticas. A su juicio, el control de constitucionalidad de las medidas del legislativo puede llegar a ser máximo cuando se aplica el control material intensivo. Su modelo de aplicación de los derechos explicita su función como límites al poder legislativo. Como dicho control de las leyes debe ser un control jurídico, el tribunal constitucional debe determinar qué ordenan, prohíben o permiten los derechos fundamentales con respecto a cada intervención legislativa. Su modelo de aplicación de los derechos les permite hacer efectiva su fuerza normativa, por lo cual, en lo relativo a la crítica política, queda claro que el control no se debe extender para derivar de los derechos órdenes, prohibiciones o permisos que sus ámbitos normativos no comprenden. Consúltese, Carlos Bernal Pulido, El principio de proporcionalidad y los derechos fundamentales. Op. cit. Sobre la racionalidad de la ponderación, véase el primer capítulo de su libro El neoconstitucionalismo y la normatividad del derecho. Op. cit. 
petrificada, lo que podría servir de máscara para legitimar una restricción a un derecho (por acción u omisión) carente de justificación.

La proporcionalidad, como criterio de interpretación judicial de las disposiciones de derecho fundamental, arroja desde la investigación de CLÉRICO una potencial línea de investigación sobre las relaciones entre el neoconstitucionalismo y la argumentación jurídica, pues como señalara AleXy en su discurso de recepción del doctorado honoris causa que recientemente le confiriera la Universidad de Alicante, "La implantación de un Estado Constitucional Democrático crea ciertamente un espacio institucional para la solución de los problemas jurídicos. Pero este espacio necesita ser llenado. El medio para ello es la argumentación jurídica”.

En este sentido una tesis que se sustenta en el libro, expresa que el examen de proporcionalidad debe interpretarse en principio como un examen formal-procedimental bajo consideración de sus tres submandatos. Formal porque no exige que su resultado corresponda a valores materiales. Se desarrolla entonces en virtud de reglas y procedimientos que se deben seguir si se pretenden reglamentar derechos cuya validez iusfundamental se evalúa. Los criterios de decisión de la concepción formal son los tres submandatos de la proporcionalidad en sentido amplio, mientras que las exigencias de fundamentación son la proporcionalidad entre la intervención a un derecho fundamental bajo consideración y la importancia del derecho o bien opuesto. En los casos difíciles dicha proporcionalidad depende enteramente de los argumentos que, en el caso puntual, pueden respaldar posiciones a favor o en contra de cada uno de los principios en colisión. De aquí la necesaria articulación entre la proporcionalidad, en particular la ponderación como su epicentro, y la argumentación.

El trabajo de CLÉRICO explora con precisión analítica y extremo rigor académico las debilidades y fortalezas de la aplicación del examen por los tribunales constitucionales en el marco de una práctica constitucional relativamente estable. Se reconstruye un conjunto amplio de reglas que pretenden delimitar el resultado del examen de proporcionalidad (como mandato de prohibición por acción excesiva y como mandato de prohibición por omisión o acción insuficiente). De esta forma se demuestra que un modelo de proporcionalidad orientado por reglas se aleja del criticado modelo de ponderaciones ad hoc donde el tribunal adquiere un amplio margen de discrecionalidad para resolver el conflicto entre derechos. Se advierte, sin embargo, que el modelo de proporcionalidad orientado por reglas requiere de una dogmática de los derechos fundamentales crítica y de tribunales superiores que se consideren a sí mismos como sujetos institucionales que hacen parte de una comunidad argumentativa abierta al debate público, pues de lo contrario este examen puede ser utilizado como una herramienta para justificar un status quo que no hace más que perpetuar severas condiciones de desigual jerarquía social. 
A la proporcionalidad en sentido amplio se ha denominado: principio, mandato, regla y criterio, así como factor y juicio. Esta amplia caracterización es criticada por Clérico. Afirma que los tres submandatos de la proporcionalidad tienen carácter de reglas y, más precisamente, reglas de segundo grado porque determinan cómo deben aplicarse las normas principiales. En sustento señala que el mandato no se pondera frente a otros mandatos sino que determina cómo deben ponderarse los objetos de la ponderación. De ahí que el título del libro no aluda entonces, como es usual en español, al principio sino al examen de proporcionalidad.

La concepción que se sigue sobre la definición y naturaleza de los principios jurídicos es la presentada por Alexy. Los principios son mandatos de optimización que ordenan que algo sea realizado en la mayor medida posible conforme a las posibilidades fácticas y jurídicas existentes. Los mandatos de idoneidad y necesidad establecen lo que es iusfundamentalmente admisible en relación con la satisfacción de un principio determinado, considerando las posibilidades fácticas; la ponderación establece la satisfacción posible del principio, considerando las posibilidades jurídicas. Conforme a CLÉRICO, determinadas las medidas posibles que revisten la misma idoneidad para contribuir al fin propuesto por la medida legislativa, el subprincipio de necesidad precisamente obliga a que la medida de intervención en el derecho fundamental sea la más benigna con el derecho intervenido.

Una idea central que resulta correlativa a esta comprensión de los principios es que los derechos fundamentales no son absolutos, que en la vida en comunidad y con un ordenamiento jurídico complejo como marco de solución de conflictos, es ineludible el conflicto entre derechos que obliga a establecer jerarquías móviles de prelación ante el caso concreto. Inevitablemente hay que articular las exigencias que se derivan de las provisiones contenidas en los derechos en conflicto. En el mismo sentido, el respeto al principio de proporcionalidad permite determinar que las intervenciones estatales que vulneran parte de las mismas son una consecuencia legítima de la democracia y válidas en un estado de derecho (social o democrático). Idea cercana a ésta, importante en la narrativa de Clérico, es el peso abstracto de los derechos como preconsideración del examen de proporcionalidad.

Críticos y detractores del canon neoconstitucional objetan instituciones jurídicas como el recurso de amparo, elementos doctrinarios como la constitución entendida como norma directamente aplicable, planteamientos teóricos como la interpretación axiológica de las provisiones que contienen los derechos y herramientas interpretativas como el principio de proporcionalidad, debido al uso que en contextos determinados se les ha dado. Aciertan las objeciones a la ponderación en sentido abstracto. Un modelo de la ponderación orientado por reglas como el que sustenta CLÉRICO, por el contrario, supone un modelo abstracto-concreto en virtud del cual se puede reconstruir 
la ponderación con la precondición de la estabilidad siquiera relativa de la práctica constitucional. La racionalidad del modelo de la ponderación se enfoca en el procedimiento de justificación de las soluciones iusfundamentales.

La crítica, que como dijo Popper es la sabia de la filosofía, no sólo no debe rechazarse sino que debe acogerse por parte de los cultores de las nuevas doctrinas sobre el constitucionalismo si quieren ser validados sus elementos medulares, pero particularmente la recepción que de ellos se ha hecho en contextos particulares. Por ejemplo, el Tribunal Constitucional Peruano acogió restringidamente el principio de proporcionalidad en sentido amplio como categoría para examinar la constitucionalidad de las leyes, pues respecto del juicio de igualdad sólo aplicaba los dos primeros subprincipios, es decir, el de idoneidad (el trato diferenciado es idóneo para contribuir a su finalidad constitucionalmente legítima) y el de necesidad (la opción del medio menos gravoso). Resulta inadmisible constitucionalmente que en el juicio de igualdad no se aplique la proporcionalidad en sentido estricto, es decir, la ponderación, pues en tal caso se podría llegar a considerar que no sería inconstitucional (que sería iusfundamentalmente justificable) una medida que afectara intensamente la igualdad para favorecer levemente un fin legítimo. A partir del 2004 el Tribunal empezó a considerar en su argumentación el tercer subprincipio de la proporcionalidad, pues precisamente la importancia de la intervención en el derecho fundamental o fin constitucional debe justificarse por la importancia de la realización del fin perseguido por la medida que interviene el derecho o fin.

CLÉRICO expone dos versiones posibles del examen de idoneidad, una fuerte y una débil, y resulta interesante que acoja la segunda. Lo primero tiene que ver con el que un medio puede ser idóneo en diferentes grados para conseguir un objetivo y la relación medio-fin puede tener mayores o menores intensidades según diversos tipos de variables, por ejemplo, desde la propia relación medio-fin, el medio elegido puede ser más o menos eficaz; desde lo temporal el medio puede contribuir más o menos con el objetivo propuesto; desde su realización, el medio puede contribuir para que se realicen más o menos aspectos relativos al fin. Sobre lo segundo, CLÉRICO defiende una versión débil del examen de idoneidad porque resulta más respetuosa con las funciones del poder legislativo en la medida en que el rasero de enjuiciamiento se vuelve de mínimos logrados y no de máximos posibles por alcanzarse. La regla argumentativa que sintetiza la concepción de este subprincipio comporta entonces la independencia del grado en el cual se hace efectiva la realización del fin por la medida bajo examen.

Si al contrario se defendiera una versión fuerte, entonces sólo una medida que contribuya de forma totalmente eficaz, rápida y segura con el fin propuesto superaría el examen de constitucionalidad, lo cual en medida significativa implica que el tribunal constitucional contraría el principio formal de 
configuración constitucional por vía legislativa. Además el examen material de constitucionalidad se reduciría porque cualquier medida legislativa que no sea la más idónea para la realización del fin, sería automáticamente declarada inconstitucional, lo cual impediría observar otras cuestiones con relevancia constitucional y que tienen relación con la proporcionalidad de las medidas, pero las cuales se suscitan sólo después, en sede de los exámenes de necesidad y en la ponderación. En Colombia, por ejemplo, de un análisis constitucional a la realización de las políticas públicas, queda claro que el ejecutivo y el legislativo son los poderes que tienen la potestad para diseñar y ejecutar las medidas con que se pretendan realizar las provisiones constitucionales, por lo cual el papel de la Corte, sin tenerse que limitar a la vigilancia inerte del cumplimiento de reglas procesales, debe ser de una máxima consideración hacia el ámbito del legislativo para evaluar las razones políticas tendientes a adoptar las medidas que considere mejores con miras a alcanzar sus objetivos ${ }^{10}$.

El mandato de proporcionalidad opera como uno de los criterios empleados para controlar la actividad de los poderes públicos que incide en la órbita de los derechos fundamentales, particularmente la que lleva a cabo el legislador en ejercicio de las competencias que la constitución le otorga para configurar, concretar o restringir tales derechos. Constituye entonces un "límite de los límites" de los derechos fundamentales. El principio de proporcionalidad cobra su mayor trascendencia cuando actúa como límite de los límites. La competencia del legislador para configurar y restringir los derechos fundamentales está limitada por cuanto el sacrificio de los derechos no puede ir más allá de lo necesario para el logro de los objetivos perseguidos por la medida que interviene el derecho. Si bien la ley es el límite de los derechos, en las democracias contemporáneas tiene lugar un control de constitucionalidad porque dicha competencia no es absoluta. En su labor de control de las intervenciones legislativas en los derechos, el juez constitucional se vale de los "límites de los límites", entre ellos del principio de proporcionalidad, para establecer si las intervenciones resultan admisibles, con lo cual los derechos aparecen como límite de la ley. El juez debe contar con herramientas para establecer hasta dónde el derecho limitado tolera las limitaciones que se le imponen, lo que resulta imperativo al momento de concretar el significado de los derechos fundamentales porque no contiene las reglas que regulan su propia aplicación.

Quisiera destacar, para concluir, un elemento del libro por el cual encontré particularmente valioso su estudio. CLÉRICO oxigena el debate constitucional

10 Al primer ámbito, al de estudiar la información técnica que se dispone para diseñar las políticas, lo denomina ALEXY "ámbito legislativo de apreciación fáctica"; al segundo, o de la adopción de las medidas efectivas para conseguir los fines propuestos, lo denomina "ámbito legislativo de apreciación normativa". Además de Clérico, siguen esta argumentación Bernal Pulido y BorowsKi. 
en un sentido valioso en la medida en que se integra a (y contribuye con) un estado del arte enriquecido por otros trabajos de singular valía académica y doctrinal sobre los elementos medulares del neoconstitucionalismo. La labor que llevan a cabo los tribunales constitucionales como guardianes de la superioridad e integridad de la constitución, que es frecuentemente criticada por la objeción contramayoritaria al poder judicial ${ }^{11}$. La agenda investigativa de Clérico señala que los esfuerzos en términos de la concepción de los derechos fundamentales, y particularmente la práctica de su aplicación, deben dirigirse -más que a la restricción del control de constitucionalidad de las leyes o a la renuncia del papel comprometido con el progresismo igualitarista de la jurisprudencia- hacia el establecimiento de límites demarcadores de la competencia del tribunal constitucional mediante la estructuración de criterios sólidos que doten de legitimidad a las decisiones de las instituciones sin representación mayoritaria.

Debido a que las normas jurídicas, por sus propios procedimientos de aprobación mediados al menos en teoría por el deliberativismo democrático, están investidas por un halo de legitimidad constitucional, los mecanismos para revertir tan privilegiada dignidad en un Estado de derecho, si no están constitucionalmente consagrados, tienen que contar con un alto grado de sofisticación teórica y de estabilidad práctica para validarse ante la sociedad. El principio de proporcionalidad en sentido amplio como el mecanismo argumentativo para fundamentar dichas invalidaciones de leyes por cuenta de su trasgresión iusfundamental, sólo será legítimo cuando gocen de diafanidad su estructura y sus propiedades conceptuales.

El ejercicio de la garantía de los derechos en un marco institucional de respeto al principio de división de poderes y de consideración a la esfera de decisión del poder legislativo, impone una serie de cuestiones concernientes con las relaciones entre la justicia constitucional y el poder legislativo. El libro no las aborda porque claramente excede su cometido investigativo. Sin embargo, se analizan en los capítulos dos y cuatro una serie de reglas para determinar el alcance de la competencia del control ante el tribunal constitucional, particularmente enfocado en la práctica del tribunal germano. Sobre tal tensión el énfasis se hace en la posibilidad de determinar con menor o mayor probabilidad el resultado del examen de idoneidad.

El carácter formal de la proporcionalidad revela su incompletitud en tanto examen que ofrece sólo una condición de legitimidad iusfundamental insuficiente; requiere una articulación con la fundamentación de la intensidad o grado de control que ejerce el tribunal constitucional sobre las medidas

11 Asunto que ha merecido singular atención en el debate iusconstitucional iberoamericano y anglosajón, norteamericano particularmente. Para una significativa muestra de los principales argumentos a favor y en contra de la misma, véanse las contribuciones de la tercera parte de El canon neoconstitucional. Op. cit. 
tomadas por el legislador en el caso concreto. CLÉRICO acentúa la naturaleza incompleta del examen de proporcionalidad como tal porque no hacerlo puede suponer una práctica legitimadora del statu quo de la práctica constitucional.

De tal acento se deriva la génesis medular del libro. Un examen meramente formal de la justificabilidad iusfundamental de una medida, ofrece una condición de legitimidad iusfundamental necesaria pero no suficiente. La suficiencia la constituiría su relación con una fundamentación de la intensidad o grado del control. La dogmática de reglas del mandato de proporcionalidad en sentido amplio que procura desarrollar el libro, complementa los submandatos de la proporcionalidad con reglas de la ponderación. El marco ideológico lo constituye la imperatividad por respetar la voluntad del legislador, pero así mismo por realizar la pretensión de efectividad que comportan los derechos una vez positivados en las constituciones del neoconstitucionalismo. Tal imperatividad exige, pues, la vinculación a reglas por parte de quien aplica el derecho en sede de revisión.

Es inversamente proporcional al margen de apreciación que se reconozca al congreso, la posibilidad de controlar el diagnóstico y pronóstico de la idoneidad del medio elegido por el legislativo, mientras que es directamente proporcional la posibilidad de que la medida no sea declarada inadecuada técnicamente, y así inconstitucional. En sentido contrario, es directamente proporcional al margen de apreciación que se le reconozca al parlamento la posibilidad de controlar los efectos del medio elegido por el congreso. Hay que tener en cuenta que claramente no implica una declaración necesaria de falta de idoneidad, es decir, de inconstitucionalidad. En un breve pero comprehensivo prólogo, ALEXY destaca precisamente que el problema de la intensidad del control en el examen de idoneidad es detallado con gran precisión. El prólogo en un primer momento despista al lector porque ALEXY alude a la versión original en alemán que consta de tres capítulos. Quizá la segunda edición podría agregar "Prologo a la versión alemana", precisando los cambios de la versión castellana en nota al pie, tal como se hace a lo largo del libro en casos de otras modificaciones de la traducción con respecto al original.

En el contexto de la concepción de los derechos fundamentales, y así de la función institucional de interpretación y aplicación, se encuentra la discusión por la proporcionalidad como herramienta que permita un control a la labor del congreso sin invadir su órbita de competencia. Adicionalmente, si se le prestara mayor atención al estado actual del derecho y a la política en los países de la región, sin duda tendríamos una perspectiva muy diferente de la "dificultad contramayoritaria" como la cuestión central del derecho constitucional $^{12}$, pues hacia otras instituciones y ramas del poder público habría que

12 Mark A. Graber. The nonmajoritarian difficulty: legislative deference to the judiciary. En 7 Stud. Am. Pol. Dev. 35. (1993). 
dirigir las críticas por la violación de derechos (por acción, omisión o acción estatal insuficiente) y la transgresión de principios (igualdad, equidad, separación de poderes, Estado social de derecho), lo cual ha desembocado en un quebrantamiento del ya débil sistema institucional en nuestra Latinoamérica.

La referida complejidad del principio de proporcionalidad por los diversos aspectos que vincula su análisis y los elementos directamente relacionados con su aplicación, se refleja en la complejidad propia de los trabajos de índole analítica que contribuyen con la sofisticación de los elementos que integran la categoría. El de ClÉRICo no es un libro oscuro en su estilo, sino exigente en su estudio debido a la minuciosidad investigativa, riqueza argumentativa, claridad analítica y rigor expositivo. Se trata de un libro para especialistas o para profesores con conocimiento en grado significativo del constitucionalismo contemporáneo, por lo que su cabal comprensión es un reto nada desdeñable. El hecho de que se analicen casi exclusivamente decisiones del tribunal constitucional federal alemán, hace que el estudioso interesado derive consecuencias para otros contextos de enseñanza del derecho y de práctica jurisprudencial, entre otros.

Impone sobre la doctrina un trabajo de pedagogía y difusión para que el principio de proporcionalidad, como es la aspiración de todas las doctrinas jurídicas, se realice en la práctica de forma consistente y sólida. La proporcionalidad, como criterio de constitucionalidad argumentativamente desarrollado por las instancias de control constitucional, al no estar tipificada expresamente en constituciones que, como la española y la alemana, son enmarcadas dentro de la fenomenología neoconstitucional, también impone ineludiblemente sobre los académicos comprometidos no sólo con la preparación de clases sino también con la mejoría de las condiciones bajo las cuales se interpreta y aplica el derecho vigente, una labor de clarificación del tipo de ente jurídico que es dicho principio y de donde recibe su validez. Su alcance de aplicación, y su misma legitimidad, dependen en gran medida, ante falta de consagración normativa, del trabajo académico. No obstante, el hecho de que el mandato de proporcionalidad no esté entonces consagrado en los textos constitucionales, y adicionalmente a lo que ello impone a la doctrina iusconstitucional, su rango constitucional ha sido reconocido por la jurisprudencia que, con fuerza argumentativa, ha estructurado con la práctica una justificación coherente de su ejercicio. 